\title{
Retracing the PAinlevé-Gambier Classification FOR DISCRETE SYSTEMS
}

\author{
B. Grammaticos and A. Ramani \\ Dedicated to Martin D. Kruskal on the occasion of his 70th birthday.
}

\begin{abstract}
We present the discrete equivalent of the Painleve list of second order differential equations which possess the Painleve property. In the discrete case, the equations we consider are three-point rational mappings. The investigation of their integrable character is based on the singularity confinement criterion. The mappings we obtain belong to three large classes in perfect analogy with the continuous case. The first class contains mappings integrable in terms of elliptic functions. The second class contains the mappings that can be reduced to linear ones. Finally, the third class consists of mappings that define discrete transcendents, i.e., the discrete analogs of the Painlevé equations.
\end{abstract}

\section{Introduction}

The integrability of low-order differential equations attracted the attention of late nineteenth and early twentieth century mathematicians. Thanks to Painlevé and his school, considerable progress has been realized in this domain but, somehow, the initial impetus gradually disappeared, and the whole integrability business faded out. Then, through one of those miracles that do occur in the par excellence rationalistic domain of mathematics and physics, the interest in integrability was revived, more than half a century later.

While investigating a model of a lattice with nonlinear interactions, Fermi, Pasta, and Ulam (FPU) [10] made an astonishing discovery: instead of energy equipartition between the various modes, the system exhibited a recurrent behavior. This indicated that some stable structure persisted in the evolution of the system. Several years later, Kruskal and Zabusky [43] investigated an equation that could be interpreted as a continuous limit of the FPU model and was known to possess a stable solitary-wave solution: the Korteweg-de Vries (KdV) equation [34]. A new astonishing discovery was made at this point: the solitary waves of $\mathrm{KdV}$ were stable even when interacting among themselves. This led Kruskal and his collaborators to conjecture (and, shortly afterwards, to prove) the integrability of the KdV equation [13]. This integrability turned out to be of an unusual type. While the integrable partial differential equations (PDEs) known at the time were linearizable through a Cole-Hopf transformation, the $\mathrm{KdV}$ equation was fundamentally different. As discovered by Kruskal et al., it could be written as the compatibility condition of an overdetermined linear system. The latter

Received October 13, 1995, revised August 8, 1996.

1991 Mathematics Subject Classification: integrability, Painlevé equations, discrete symmetry.

Key words and phrases: 35Q53, 58F01, 35Q58, 39A10, 65Q05. 
was subsequently called the "Lax pair" [27], a notion which became a cornerstone of the modern approach to integrability. The crucial step came with the realization that, given the Lax pair, the techniques of inverse scattering (for the reconstruction of the potential from scattering data) could be used in order to solve the initial-value problem for $\mathrm{KdV}$. This discovery rekindled the interest in integrable systems, an interest that has not faded away more than a quarter century later.

It was natural in this framework of integrable systems to come back to the question of the Painlevé equations. Painlevé [32] had, in fact, studied second-order differential equations of the form $w^{\prime \prime}=f\left(w^{\prime}, w, z\right)$ where $f$ is rational in $w^{\prime}$, algebraic in $w$, and analytic in $z$, from the point of view of the singularity structure of their solutions. He had, with the help of Gambier [12], identified fifty equations the solutions of which did not contain movable critical singularities. They have shown that the majority of these equations were integrable (or reducible to some other, simpler, equation among the fifty). However, six of these equations could not be integrated with the techniques available at that time. Painlevé's intuition led him to decide that they were integrable indeed, defining new transcendents. The derivation of "Lax pairs" for the Painlevé equations did not take too long. Garnier [14] derived the linear systems that led, as compatibility conditions, to the Painlevé equations, but did not use them in order to integrate the latter. This was done for the first time by Ablowitz and Segur [4], using inverse scattering techniques. They have shown in fact that the linearization of the Painlevé equations can be realized through integrodifferential equations.

Ablowitz and Segur (in close collaboration with Kruskal) [3], and also Hastings and McLeod [22], made another observation. All the reductions of integrable PDEs that could be written as second-order ordinary differential equations (ODEs) were members of the Painlevé-Gambier list. This led naturally to the formulation in collaboration with one of us (Ramani) of the ARS conjecture [2]: "Every nonlinear ODE obtained by an exact reduction of a nonlinear PDE solvable by inverse scattering techniques is of P-type". This means that all its solutions have the Painlevé property, i.e., they are free of movable critical singularities. They also developed an efficient algorithm for the investigation of the Painlevé property. This led to a whole branch in the domain of integrability [38] and, what is perhaps even more important, it forged a tool, both efficient and easy to use, for the study of integrability.

While continuous systems were in full development, the situation was quite different for discrete systems. One of the reasons (perhaps the main one) was that there was, at the time, not much "physical" interest in discrete systems. The modeling of the various physical phenomena proceeded usually through continuous systems, and on those occasions where discrete systems were considered, this was done solely in order to study their chaotic behavior. Another, non-negligible, point was the fact that discrete systems are, a priori, much more difficult to study than continuous ones. (This last statement is to be understood in the situation of the 70 s and early 80 s when the tools for the study of discrete systems had not yet been developed). Isolated efforts, of course, did lead to very interesting results in the domain of discrete integrability. We can mention here the works of Ablowitz and Ladik [1], Hirota [23], and Capel and collaborators [35], but, on the whole, those attempts did not manage to create an impetus in the right direction. What also was lacking was the equivalent of the ARS-Painlevé approach: an integrability detector for discrete systems. The latter had to wait till the $90 \mathrm{~s}$. At that time, by a happy coincidence that occurs frequently in 
scientific research, the conditions became right for the derivation of a discrete integrability detector. (Note that "coincidence" is perhaps not the right word. What really happens is a collective phenomenon whereby a critical mass is reached and the proper impetus is maintained). First, recent work in field theory had turned the interest towards discrete systems [6]: physicists started realizing that discrete systems were more fundamental than continuous ones. Second, integrable discrete systems had started appearing [31], replacing the previous scarcity by a body of examples that would allow the formulation and testing of conjectures on integrability. It was in this context that we proposed [20] the "singularity confinement" method, that has (four years later) proven beyond doubt its efficiency as an integrability detector. The principle is in fact simple. In a rational mapping, singularities may appear spontaneously due to a particular choice of initial condition. In analogy to the continuous case, we call these singularities "movable". Our conjecture states that, in integrable systems, these singularities must disappear after a few iterations. This is what is meant by "confinement". The implementation of this method has been reviewed in detail in [41]. We shall limit ourselves here to probably the most important application of the singularity confinement method: the derivation of the discrete Painlevé equations.

As was mentioned above, Painlevé and Gambier accomplished the difficult task of classifying all the integrable second-order equations of the form $w^{\prime \prime}=f\left(w^{\prime}, w, z\right)$. These equations turned out to be of the utmost usefulness in the study of physical problems. It was thus natural to try to establish the analogous results in the case of discrete equations. Discrete Painlevé equations had already made their appearance in the studies related to the Ising model [25, 28], and, in fact, the first reference remarked on the relation of the discrete equation obtained and Painlevé equations. Similarly, in a work of Jimbo, Miwa, and Ueno [26] going back to 1981, a discrete Painlevé equation appears (it is the "alternate" $d-P_{I}$ of [11]), but its analogy to the continuous Painlevé equation was not established. A more recent and unambiguous reference to a discrete Painlevé equation is made in the work of Brézin and Kazakov [6] who, studying a two-dimensional model of quantum gravity, obtained the 'standard' form of d-P $P_{I}$. Shortly afterwards, d-P $P_{\text {II }}$ was also obtained $[30,33]$, and the introduction of singularity confinement allowed us to derive [39] the remaining d-Ps up to d-P $\mathrm{P}_{\mathrm{V}}$. Since this first work, many more results have been obtained, and today we can show that the whole Painlevé-Gambier classification can be retraced in a discrete setting. Before proceeding further, we must point out that our approach is not the same as that of Painlevé and Gambier. Those authors investigated systematically (Painlevé with his " $\alpha$-method" while Gambier used Kovalevskaya's approach) second-order ODEs and identified those which satisfied the integrability criterion. In our approach, we do not implement singularity confinement in the most general discrete system but, rather, look for the discrete analogs of the equations of the Painlevé-Gambier list. Thus our search is guided by the results of the continuous case, and the singularity confinement criterion is used in order to ensure integrability of the forms obtained.

The discrete equivalent to the second-order differential equations of the Painlevé family we are going to study here is the three-point explicit rational mapping. It is clear that this is not the only possibility. However, it is a most natural one and as we shall show, it is rich enough to yield the expected results. Before examining the three-point case, we shall start with two-point mappings which are equivalent to first-order differential equations. 


\section{First-order equations and two-point mappings}

The study of integrable differential equations was initiated by Fuchs who studied firstorder equations, (algebraic in $w, w^{\prime}$ ) the solutions of which have fixed critical points. Poincaré has shown that these equations either have all their solutions algebraic, or are algebraically reducible to a quadrature, or are of Riccati form. If we focus on equations explicit in the first derivative, $w^{\prime}=f(w, z)$, the only first-order equations that have what later became known as the Painlevé property are

$$
\begin{aligned}
& w^{\prime}=a w+b, \\
& w^{\prime}=-w^{2}+a w+b .
\end{aligned}
$$

It is instructive to analyze the first-order equations in the light of modern singularity analysis. Let us start with a first-order equation $w^{\prime}=f(w, z)$ where $f$ is a polynomial in $w$ of degree $k$. Then the dominant singular behavior of the solutions of the equations is $w \propto\left(z-z_{0}\right)^{-1 /(k-1)}$. Thus, except for the Riccati case $k=2$, the singularity presents a branching incompatible with the Painlevé property.

What are the discrete equivalents of equations (2.1) and (2.2)? Clearly, the linear map

$$
x_{n+1}=a x_{n}+b
$$

is the discrete analog of (2.1). In the case of the Riccati equations, the discretization of the derivative does not cause any difficulty: a finite difference $x_{n+1}-x_{n}$ suffices. The difficulty lies in the discretization of the nonlinear term. To make a long story short [40], the only discretization that leads to an integrable mapping is to replace $w^{2}$ by $x_{n+1} x_{n}$. Thus, the discrete Riccati equation is the homographic mapping

$$
x_{n+1}=\frac{a x_{n}+b}{c x_{n}+d} .
$$

Using scaling, we can bring (2.4) to the form $x_{n+1}-x_{n}=\left(-x_{n}^{2}+a x_{n}+b\right) /\left(1-x_{n}\right)$, a form that clearly evokes (2.2). The parallel between the continuous and the discrete Riccati equations is perfect. Both are linearizable through a Cole-Hopf transformation. Putting $w=P / Q$, or $x=P / Q$, allows one to reduce them to a linear equation of second order or to a linear three-point mapping, respectively.

What does singularity confinement tell us about two-point mappings? Let us consider in general $x_{n+1}=f\left(x_{n}, n\right)$ where $f$ is rational in $x_{n}$. As was shown in [40], the only form that satisfies the singularity requirement is

$$
f\left(x_{n}, n\right)=\alpha+\sum_{k} \frac{1}{\left(x_{n}-\beta_{k}\right)^{\nu_{k}}}
$$

with positive integers $\nu_{k}$, provided that for all $k, \beta_{k} \neq \alpha$. Indeed, if $x_{n}=\beta_{k}$ at some step, then $x_{n+1}$ diverges, $x_{n+2}=\alpha$, and $x_{n+3}$ is finite. So the mapping propagates without any further difficulty. However, if we consider the "backward" evolution, then (2.5) solved for $x_{n}$ in terms of $x_{n+1}$ leads to multideterminacy, and the number of preimages grows exponentially with the number of 'backward' iterations. Indeed, the only mapping of this form with no growth is just the homographic (2.4).

The argument of non-proliferation of preimages [21] is essential for the integrability of discrete systems. In fact, in every case that we consider, this no-growth criterion 
is implemented even before one starts examining the possible singularities and their confinement.

\section{The second-order (Painlevé/Gambier) equations and the corresponding three-point mappings}

The aim of this paper is to establish the discrete analog of the Painlevé/Gambier classification of integrable second-order differential equations. Before embarking upon examining the discrete forms, one must establish clearly which are the continuous equations one wishes to discretize. The common lore is that there exist 50 canonical equations of the form

$$
w^{\prime \prime}=f\left(w^{\prime}, w, z\right)
$$

(with $f$ rational in $w^{\prime}$, algebraic in $w$, and analytic in $z$ ) that possess the Painlevé property. They were first compiled by Gambier [12] and canonized through the book of Ince [24]. Recently, Cosgrove [9] raised objections as to the canonical nature of this list and, in a work that is (unfortunately) still part of the apocrypha of integrability, talked about 74, 81, or even 120 canonical forms. (In fact, Gambier himself has made clear that the number of possible forms of the equations of the form (3.1) ranges well into the hundreds). So, had it not been for Gambier, we would be faced with a hard task.

Fortunately, in his seminal work, Gambier presented a table of 24 equations that he considered as fundamental. In fact, Gambier constructs "... un tableau $(t)$, dont il suffit d'indiquer la nature de chaque équation pour en déduire immédiatement la nature de toutes les équations $T$ et en donner l'intégration effective, ..." ". In other words, determining the solutions of the 24 equations of his table $(t)$ would allow one to find the solutions of all the equations of the larger list $(T)$, which are the 50 listed by Ince, and through a homographic transformation of the dependent variable, plus a transformation of the independent variable, the solutions of all the equations of form (3.1) with the Painlevé property.

Here is the Gambier $(t)$ list, where $a, b, c, d, e$ are constant, $q$ and $r$ are free functions of $z$, and $f_{n}, \phi_{n}$, and $\psi_{n}$ are given functions of $q$ and $r$ :

$$
\begin{aligned}
& w^{\prime \prime}=0, \\
& w^{\prime \prime}=6 w^{2}, \\
& w^{\prime \prime}=6 w^{2}-1 / 24, \\
& w^{\prime \prime}=6 w^{2}+z \\
& w^{\prime \prime}=-3 w w^{\prime}-w^{3}+q\left(w^{\prime}+w^{2}\right), \\
& w^{\prime \prime}=-2 w w^{\prime}+q w^{\prime}+q^{\prime} w, \\
& w^{\prime \prime}=2 w^{3}, \\
& w^{\prime \prime}=2 w^{3}+a w+b, \\
& w^{\prime \prime}=2 w^{3}+z w+a,
\end{aligned}
$$




$$
\begin{aligned}
& w^{\prime \prime}=\frac{w^{\prime 2}}{w} \\
& w^{\prime \prime}=\frac{w^{\prime 2}}{w}+a w^{3}+b w^{2}+c+\frac{d}{w}, \\
& w^{\prime \prime}=\frac{w^{\prime 2}}{w}-\frac{w^{\prime}}{z}+\frac{1}{z}\left(a w^{2}+b\right)+c w^{3}+\frac{d}{w}, \\
& w^{\prime \prime}=\frac{w^{\prime 2}}{w}+q \frac{w^{\prime}}{w}-q^{\prime}+r w w^{\prime}+r^{\prime} w^{2}, \\
& w^{\prime \prime}=\left(1-\frac{1}{n}\right) \frac{w^{\prime 2}}{w}+q w w^{\prime}-\frac{n q^{2}}{(n+2)^{2}} w^{3}+\frac{n q^{\prime}}{n+2} w^{2}, \\
& w^{\prime \prime}=\left(1-\frac{1}{n}\right) \frac{w^{\prime 2}}{w}+\left(f_{n} w+\phi_{n}-\frac{n-2}{n w}\right) w^{\prime} \\
& -\frac{n f_{n}^{2}}{(n+2)^{2}} w^{3}+\frac{n\left(f_{n}^{\prime}-f_{n} \phi_{n}\right)}{n+2} w^{2}+\psi_{n} w-\phi_{n}-\frac{1}{n w}, \\
& w^{\prime \prime}=\frac{w^{\prime 2}}{2 w}+\frac{3 w^{3}}{2} \\
& w^{\prime \prime}=\frac{w^{\prime 2}}{2 w}+\frac{3 w^{3}}{2}+4 a w^{2}+2 b w-\frac{c^{2}}{2 w}, \\
& w^{\prime \prime}=\frac{w^{\prime 2}}{2 w}+\frac{3 w^{3}}{2}+4 z w^{2}+2\left(z^{2}-a\right) w-\frac{b^{2}}{2 w}, \\
& w^{\prime \prime}=\frac{w^{\prime 2}-1}{2 w}, \\
& w^{\prime \prime}=w^{\prime 2}\left(\frac{1}{2 w}+\frac{1}{w-1}\right) \\
& w^{\prime \prime}={w^{\prime}}^{2}\left(\frac{1}{2 w}+\frac{1}{w-1}\right)+(w-1)^{2}\left(a w+\frac{b}{w}\right)+c w+\frac{d w}{w-1}, \\
& w^{\prime \prime}={w^{\prime}}^{2}\left(\frac{1}{2 w}+\frac{1}{w-1}\right)-\frac{w^{\prime}}{z}+\frac{(w-1)^{2}}{z^{2}}\left(a w+\frac{b}{w}\right)+c \frac{w}{z}+\frac{d w(w+1)}{w-1}, \\
& w^{\prime \prime}=w^{\prime 2}\left(\frac{1}{w}+\frac{1}{w-1}+\frac{1}{w-a}\right) \\
& +w(w-1)(w-a)\left(b+\frac{c}{w^{2}}+\frac{d}{(w-1)^{2}}+\frac{e}{(w-a)^{2}}\right), \\
& w^{\prime \prime}={w^{\prime}}^{2}\left(\frac{1}{w}+\frac{1}{w-1}+\frac{1}{w-z}\right)-w^{\prime}\left(\frac{1}{z}+\frac{1}{z-1}+\frac{1}{w-z}\right) \\
& +\frac{w(w-1)(w-z)}{2 z^{2}(z-1)^{2}}\left(a-\frac{b z}{w^{2}}+c \frac{z-1}{(w-1)^{2}}+\frac{(d-1) z(z-1)}{(w-z)^{2}}\right) .
\end{aligned}
$$


First, let us remark that two of these equations, namely (G1) and (G6) are just the derivatives of the equations we encountered at first-order. Equation (G1) is obviously the derivative of a linear equation, and (G6) is the derivative of a Riccati equation.

The remaining equations can be regrouped into three classes corresponding to the method of integration. The first class contains equations $(2,3,7,8,10,11,16,17,19$, $20,21,23)$. They are integrable through quadratures in terms of elliptic functions, and the generic equation of this class is equation (G23). The second class contains equations $(5,13,14,15)$ that can be linearized. All of them are generalizations of the Riccati equation in some sense, and equation (G15) which is due to Gambier is the generic one of this class. Finally, the third class $(4,9,12,18,22,24)$ corresponds to the transcendental equations of Painlevé, $\mathrm{P}_{\mathrm{I}}$ to $\mathrm{P}_{\mathrm{VI}}$. These equations are not integrable through quadratures, nor are they reducible to a linear differential system. Rather, their integration is obtained through IST techniques which reduce them to linear integrodifferential equations. The generic equation is $\mathrm{P}_{\mathrm{VI}}$ (G24): it can be reduced to the other transcendents through the appropriate limits.

Let us now turn to the discrete forms of these equations. Concerning the derivatives of the linear and Riccati equations, the discrete form is straightforward. We find, after several simplifications in the case of the discrete Riccati equation

$$
\begin{gathered}
x_{n+1}+x_{n-1}=a x_{n}+b, \\
x_{n+1}=x_{n-1}+a_{n}-\frac{a_{n-1} x_{n-1}}{x_{n}} .
\end{gathered}
$$

The remaining three classes have their discrete equivalent in perfect analogy to the continuous result. Let us examine the class of equations solvable in terms of elliptic functions first.

3.1. The elliptic-solutions mappings. The elliptic-solutions mappings have been analyzed in [36] where the general form has been given. The QRT form we are going to work with is

$$
x_{n+1}=\frac{f_{1}\left(x_{n}\right)-x_{n-1} f_{2}\left(x_{n}\right)}{f_{2}\left(x_{n}\right)-x_{n-1} f_{3}\left(x_{n}\right)}
$$

where the $f_{i}$ s are quartic polynomials obtained in the following way. First, one defines two symmetric matrices $A_{0}$ and $A_{1}$ of the form

$$
A_{i}=\left(\begin{array}{ccc}
\alpha_{i} & \beta_{i} & \gamma_{i} \\
\beta_{i} & \epsilon_{i} & \zeta_{i} \\
\gamma_{i} & \zeta_{i} & \mu_{i}
\end{array}\right)
$$

and the vector $\vec{X}=\left(\begin{array}{lll}x^{2} & x & 1\end{array}\right)^{T}$. We obtain next $\vec{V}=A_{0} \vec{X}$ and $\vec{W}=A_{1} \vec{X}$ and compute $\vec{F}=\left(\begin{array}{lll}f_{1} & f_{2} & f_{3}\end{array}\right)^{T}$ through $\vec{F}=\vec{V} \times \vec{W}$.

Clearly, replacing $A_{0}, A_{1}$ by two independent linear combinations of $A_{0}, A_{1}$ will leave the final result unchanged. It then always is possible to take $\alpha_{1}=0$, and we, generically, can put $\alpha_{0}=1$. (Non-generic cases, of course, may exist corresponding to $\alpha_{0}=0$ ). Moreover, we always can take $\mu_{0}=0$ and, generically, $\mu_{1}=1$. Thus, from the 12 parameters that were present in the QRT parametrization, only 8 survive at this stage. Next, from the form of the mapping, it is clear that it is invariant under homographic transformations of $x$. Since a homographic transformation has three free parameters, one expects that their use will further help to simplify the mapping 
by fixing three of the 8 remaining QRT parameters, bringing the result down to 5 . These transformations tend to be particularly intricate if performed directly. However, singularity confinement can be of great help at this point. As we have explained in [40] (see also [37]), the conditions for the appearance of a singularity of the QRT mapping (3.4) is

$$
f_{1}\left(x^{(i)}\right) f_{3}\left(x^{(i)}\right)-f_{2}^{2}\left(x^{(i)}\right)=0 .
$$

This means that there exist 8 zeroes $x^{(i)}$ 's of (3.6) that lead to a singularity (loss of a degree of freedom). If $x_{n}=x^{(i)}$, then $x_{n+1}$ is independent of $x_{n-1}$ and its value is given by

$$
y^{(i)}=\frac{f_{1}\left(x^{(i)}\right)}{f_{2}\left(x^{(i)}\right)}=\frac{f_{2}\left(x^{(i)}\right)}{f_{3}\left(x^{(i)}\right)} .
$$

The confinement of the singularity, with the assumption of a generic behavior, is realized in a single step. This means (since the QRT mapping, being integrable, must be confining) that each of the $y^{(i)}$ 's calculated above is one of the roots $x^{(j)}$ in order to lead to an indeterminate form $0 / 0$ and recovery of the lost degree of freedom. Thus, $x^{(i)}$ gives $y^{(i)}$ through (3.7), which is equal to $x^{(j)}$ for some $j$. It turns out that the specific form of the QRT map ensures that $y^{(j)}$ is precisely $x^{(i)}$. Thus, the 8 roots of (3.6) are grouped into four pairs. One enters the singularity through $x^{(i)}$ and exits through, say, $x^{(i+4)}$. Once the roots are assigned in pairs, one can perform the homographic transformation to bring three of the roots ( $\operatorname{say} x^{(1)}, x^{(2)}$, and $x^{(5)}$ ) to the values 0,1 , and $\infty$ respectively. Putting

$$
X=\frac{x-x^{(1)}}{x-x^{(5)}} \frac{x^{(2)}-x^{(5)}}{x^{(2)}-x^{(1)}}
$$

does just that. In fact, it is possible to go further and express all the parameters of the QRT mapping in terms of the remaining $x^{(i)}$ 's. As we have verified in [37], the specific QRT mapping is the only one of the form (3.4) with the polynomials $f_{i} \mathrm{~s}$ of degree less than 6 that satisfies the singularity confinement criterion. We are not going to go through this analysis, nor give the cumbersome final expressions. It is perhaps more interesting to display a case of the QRT mapping which goes over to (G23) in the continuous limit $(\varepsilon \rightarrow 0)$. The corresponding matrices are:

$$
A_{0}=\left(\begin{array}{ccc}
-2 \varepsilon^{2} b & 0 & 1 \\
0 & \epsilon_{0} & \zeta_{0} \\
1 & \zeta_{0} & 2 \varepsilon^{2} c a
\end{array}\right), \quad A_{1}=\left(\begin{array}{ccc}
0 & 1 & 0 \\
1 & -2(a+1) & a \\
0 & a & 0
\end{array}\right)
$$

with $\epsilon_{0}=2\left(\varepsilon^{2}\left(e+d+c+b\left(a^{2}+a+1\right)\right)-1\right)$ and $\zeta_{0}=-\varepsilon^{2}(e+a d+$ $(a+1)(c+a b))$. Although not singled out as a distinct equation in Gambier's list, one case is particularly interesting since it leads to a very simple expression. Taking $b=c=d=e=0$ in (G23) leads to the equation $w^{\prime \prime}=w^{\prime 2}\left(\frac{1}{w}+\frac{1}{w-1}+\frac{1}{w-a}\right)$. We can easily show that the discrete form of this equation is just

$$
x_{n+1}+x_{n-1}-2 x_{n}=\frac{\left(x_{n+1}-x_{n}\right)\left(x_{n}-x_{n-1}\right)}{2}\left(\frac{1}{x_{n}}+\frac{1}{x_{n}-1}+\frac{1}{x_{n}-a}\right) \text {. }
$$


As was shown in [36], the QRT mapping is integrable. Its first integral is given by the correspondence

$$
\begin{aligned}
& \left(\alpha_{0}+K \alpha_{1}\right) x_{n+1}^{2} x_{n}^{2}+\left(\beta_{0}+K \beta_{1}\right) x_{n+1} x_{n}\left(x_{n+1}+x_{n}\right) \\
& +\left(\gamma_{0}+K \gamma_{1}\right)\left(x_{n+1}^{2}+x_{n}^{2}\right)+\left(\epsilon_{0}+K \epsilon_{1}\right) x_{n+1} x_{n} \\
& +\left(\zeta_{0}+K \zeta_{1}\right)\left(x_{n+1}+x_{n}\right)+\left(\mu_{0}+K \mu_{1}\right)=0
\end{aligned}
$$

where $K$ is the integration constant. Expression (3.10) further can be parametrized in terms of elliptic functions [5]. This means that the solution of (3.10) is just a sampling of an elliptic function over a discretized mesh, i.e., the solution is essentially the same in the continuous and discrete cases. As we shall see in what follows, this will not be the case for the remaining Painlevé-Gambier mappings.

Once the generic mapping corresponding to (G23) is derived, it is easy to obtain the nongeneric forms corresponding to the remaining equations of this class. We shall not go into this elementary exercise, but rather proceed to the second class, that of linearizable mappings.

3.2. The linearizable mappings. In [40], we have presented a generalization of the QRT mapping that makes possible the description of linearizable mappings. This generalization reads

$$
x_{n+1}=\frac{f_{1}\left(x_{n}\right)-x_{n-1} f_{2}\left(x_{n}\right)}{f_{4}\left(x_{n}\right)-x_{n-1} f_{3}\left(x_{n}\right)}
$$

where the $f_{i}$ s are polynomials in $x$ and with coefficients that depend explicitly on $n$. In fact, just as in the case of the Painlevé-Gambier equations, the linearizable mappings are the only ones for which the $n$-dependence is not strictly fixed but, rather, can be written in terms of free functions.

Instead of analyzing (3.11) in its full generality, we shall limit ourselves to the forms that are the discrete analogs of the linearizable equations of the Gambier list. The generic mapping of this kind, corresponding to Gambier's equation (G15), is given by the coupling of a mapping $x_{n+1}$ homographic in $x_{n}$ (discrete Riccati equation) with coefficients linear in $y_{n}$

$$
\alpha x_{n+1} x_{n} y_{n}+\beta x_{n+1} x_{n}+\gamma x_{n+1} y_{n}+\delta x_{n} y_{n}+\epsilon x_{n}+\zeta y_{n}+\eta x_{n+1}+\theta=0
$$

with a discrete Riccati equation for $y_{n}$

$$
y_{n+1}=\frac{\kappa y_{n}+\lambda}{\mu y_{n}+\nu}
$$

Eliminating $y$ between (3.12) and (3.13), we find for $x$ a mapping of the form (3.11) with $f_{i}$ s quadratic in $x_{n}$. (However, this mapping is not the most general one of this form.) Since the $f_{i}$ s are quadratic the singularity condition

$$
f_{1}\left(x^{(i)}\right) f_{3}\left(x^{(i)}\right)-f_{2}\left(x^{(i)}\right) f_{4}\left(x^{(i)}\right)=0
$$

has four roots $x^{(1)}, x^{(2)}, x^{(3)}$, and $x^{(4)}$ at each $n$. As explained in [17], due to the cascade-Riccati structure of the mapping, the basic singularity pattern corresponding to this equation is the following. When $x$ becomes $x^{(1)}$ at step $n$, the mapping loses one degree of freedom and recovers it at the very next step through the value $x^{(2)}$ at step $n+1$. The same applies to the pair $x^{(3)}$ and $x^{(4)}$. These are not the only singularities of the mapping. But they will suffice in order to give the general form. 
Next, we use the invariance of (3.12) under a homographic transformation at each $n$ and assign to three of the $x_{i}$ specific values, say, $x^{(1)}=0, x^{(2)}=1$, and $x^{(4)}=\infty$.

We find that the two singularity patterns $\{0,1\}$ and $\left\{x^{(3)}, \infty\right\}$ are realized only if the mapping has the form (taking generically $\eta=1$ )

$$
\beta x_{n+1} x_{n}+x_{n+1}-1+x_{n} \frac{\delta y_{n} \epsilon}{\gamma y_{n}+1}=0 .
$$

Using the homographic freedom for $y$, we can bring (3.15) to the form

$$
x_{n+1}=\frac{1+x_{n} y_{n}}{1+\beta x_{n}} \text {. }
$$

This is precisely the form studied in [17] where we analyzed the Gambier mapping

$$
\begin{aligned}
& y_{n+1}=\frac{b y_{n}+c}{y_{n}+1} \\
& x_{n+1}=\frac{d x_{n} y_{n}+\sigma}{1-a x_{n}}
\end{aligned}
$$

where $\sigma=0$ or 1 . The coefficients, $a(n), b(n), c(n)$, and $d(n)$ are not all free. They are constrained by the singularity confinement requirement related to singularity patterns where one first encounters $x^{(2)}$ and, $N$ steps later, $x^{(1)}$ or one first encounters $x^{(4)}$ and, $N^{\prime}$ steps later, $x^{(3)}$.

We shall not go into these technical details here. It suffices to say that equation (3.17) is indeed the discrete form of Gambier's equation. Like the latter, it is built up from two Riccati equations in cascade, and indeed its continuous limit is equation (G15). Just as (G5, G13, G14) can be obtained as special limits of the Gambier equation (G15), in the same way, their discrete analogs are obtained from (3.17). Let us start with (G5). Taking $a=b=0$ (together with $\sigma=1$ ), we find

$$
x_{n+1}=\frac{x_{n-1} d(n-1)\left(c(n-1) d(n) x_{n}+1\right)+x_{n}-1}{x_{n-1} d(n-1)+x_{n}-1} .
$$

Putting $u=-1+1 / x$ we obtain

$$
u_{n-1}=\frac{u_{n+1}\left(p u_{n}+q\right)+r}{u_{n+1} u_{n}}
$$

where $r=c(n-1) d(n) d(n-1), p=d(n-1)-1$, and $q=d(n-1)(1+c(n) d(n-1))$. The mapping (3.19) is precisely the one obtained in [40] where we investigated the 3point mappings linearizable through a Cole-Hopf-like transformation. The remaining two discrete equations are obtained in a straightforward way. In the continuous case, they correspond to one of the two Riccati equations becoming linear. Equation (G13) is in fact a derivative of the Riccati equation (but different from (G6)). In the discrete case, we find the system (where, because we now have an extra freedom, $b$ can be scaled to 1)

$$
\begin{aligned}
& y_{n+1}=y_{n}+c \\
& x_{n+1}=\frac{d x_{n} y_{n}+\sigma}{1-a x_{n}},
\end{aligned}
$$

or an equivalent form in terms of $x$ alone. 
The discrete equivalent of equation (G14) corresponds to taking $\sigma=0$, in which case $(3.20 \mathrm{~b})$ becomes linear in $1 / x$ :

$$
\begin{aligned}
y_{n+1} & =\frac{b y_{n}+c}{y_{n}+1}, \\
\frac{1}{x_{n+1}} & =\frac{1}{d y_{n}}\left(\frac{1}{x_{n}}-a\right) .
\end{aligned}
$$

Thus we have established a discrete equivalent for each of the equations of the linearizable class.

3.3. The discrete Painlevé equations. As we explained in the introduction, it was the discovery of the discrete analogs of the Painlevé transcendental equations that spurred the activity around integrable discrete systems. The first discrete Painlevé equation to be acknowledged as such is $\mathrm{d}-\mathrm{P}_{\mathrm{I}}$ in the form

$$
x_{n+1}+x_{n-1}+x_{n}=\frac{z}{x_{n}}+a
$$

where $z=\alpha n+\beta$, and $a$ is a constant. The second transcendent $\mathrm{d}-\mathrm{P}_{\mathrm{II}}$ made its appearance shortly afterwards [30]

$$
x_{n+1}+x_{n-1}=\frac{z x_{n}+a}{1-x_{n}^{2}} .
$$

It is interesting to rewrite (3.23) so as to make the discretization of the second derivative appear explicitly on the left-hand side [15]. We find

$$
x_{n+1}+x_{n-1}-2 x_{n}=\frac{2 x_{n}^{3}+z x_{n}+a}{1-x_{n}^{2}} .
$$

One recognizes in the numerator of the right-hand side the non-derivative part of $\mathrm{P}_{\mathrm{II}}$. Thus the continuous limit of (3.24) is obtained by putting $x=\epsilon w$, and letting $\epsilon$ go to zero. In fact, $\mathrm{d}-\mathrm{P}_{\mathrm{I}}$ can be written in an analogous way, using a redefinition of $x$ and $z$. (For the continuous limit to exist, we must take $a \neq 0$, which can be scaled to $a=-6$ ). We find in this case (with $x \rightarrow 1-x$ )

$$
x_{n+1}+x_{n-1}-2 x_{n}=\frac{3 x_{n}^{2}+z}{1-x_{n}} \text {. }
$$

The higher discrete Ps were obtained in this same way. The idea is to start from the QRT mapping, which can be rewritten as

$$
f_{3}\left(x_{n}\right) x_{n+1} x_{n-1}-f_{2}\left(x_{n}\right)\left(x_{n+1}+x_{n-1}\right)+f_{1}\left(x_{n}\right)=0
$$

and obtain information on the form of the $f_{i}$ s, based on the continuous limit. In fact, introducing the lattice parameter $\epsilon$, we have

$$
\begin{aligned}
x_{n+1}+x_{n-1} & =2 x+\epsilon^{2} x^{\prime \prime}+\mathcal{O}\left(\epsilon^{4}\right), \\
x_{n+1} x_{n-1} & =x^{2}+\epsilon^{2}\left(x x^{\prime \prime}-x^{\prime 2}\right)+\mathcal{O}\left(\epsilon^{4}\right),
\end{aligned}
$$

and the continuous limit of (3.26) will come out as

$$
x^{\prime \prime}=\frac{f_{3}(x)}{x f_{3}(x)-f_{2}(x)} x^{2}+g(x) .
$$


Thus, when we aim at a specific d-P, we start by choosing the $f_{i}$ s in such a way as to get $f_{3}(x) /\left(x f_{3}(x)-f_{2}(x)\right)$ to coincide with the factor multiplying ${x^{\prime}}^{2}$ in the continuous analog of the desired equation.

We have thus obtained for $d-P_{\text {III }}$

$$
x_{n+1} x_{n-1}=\frac{a b\left(x_{n}-p\right)\left(x_{n}-q\right)}{\left(x_{n}-a\right)\left(x_{n}-b\right)}
$$

where $p=p_{0} \lambda^{n}, q=q_{0} \lambda^{n}$, and $a, b$ are constants. Let us point out that this form of d-P $\mathrm{P}_{\text {III }}$ corresponds to a $\mathrm{P}_{\text {III }}$ obtained from (G12) through the transformation of the independent variable $z \rightarrow Z=e^{z}$; this transformation allows us to eliminate the term linear in $w^{\prime}$. (The same transformation will be used in the case of $d-P_{V}$.) As we have shown in [16], the discrete form (G12) also can be derived directly. It corresponds to a system of two two-point mappings

$$
\begin{aligned}
x_{n+1}+x_{n} & =\frac{z(n) y_{n}+a}{y_{n}^{2}-1} \\
y_{n}+y_{n-1} & =\frac{z(n-1 / 2) x_{n}+b}{x_{n}^{2}-1} .
\end{aligned}
$$

For d- $\mathrm{P}_{\mathrm{IV}}$ and $\mathrm{d}-\mathrm{P}_{\mathrm{V}}$, we can give analogous expressions $(a, b, c$ are constants)

$$
\begin{aligned}
\left(x_{n+1}+x_{n}\right)\left(x_{n}+x_{n-1}\right) & =\frac{\left(x_{n}^{2}-a^{2}\right)\left(x_{n}^{2}-b^{2}\right)}{\left(x_{n}-z\right)^{2}-c^{2}}, \\
\left(x_{n+1} x_{n}-1\right)\left(x_{n} x_{n-1}-1\right) & =\frac{p q\left(x_{n}-a\right)\left(x_{n}-1 / a\right)\left(x_{n}-b\right)\left(x_{n}-1 / b\right)}{\left(x_{n}-p\right)\left(x_{n}-q\right)} .
\end{aligned}
$$

In the case of $\mathrm{d}-\mathrm{P}_{\mathrm{V}}$, the continuous limit of (3.32) is not (G22), but rather a more symmetric form obtained from (G22) through a transformation of the dependent variable $u=\frac{w+1}{w-1}$ (in addition to the transformation of the independent variable we mentioned above). In this case, the coefficient of ${u^{\prime}}^{2}$ is $\frac{1}{2}\left(\frac{1}{u-1}+\frac{1}{u+1}\right)$.

Just as in the continuous case, the d-Ps above are organized in a coalescence cascade in the form:

$$
\mathrm{d}-\mathrm{P}_{\mathrm{V}} \rightarrow\left\{\mathrm{d}-\mathrm{P}_{\mathrm{IV}}, \mathrm{d}-\mathrm{P}_{\mathrm{III}}\right\} \rightarrow \mathrm{d}-\mathrm{P}_{\mathrm{II}} \rightarrow \mathrm{d}-\mathrm{P}_{\mathrm{I}} .
$$

Contrary to the continuous case, the canonical forms of the d-Ps are not unique. Several possible forms do exist for each equation, all of them having the appropriate continuous limit. Each of these equations, of course, has all the analogs of the properties that characterize the corresponding continuous equation. These properties have been the object of intense study these last few years. In particular, the discrete Painlevé equations [18]

- form coalescence cascades,

- possess Lax pairs,

- have solutions related through Bäcklund and Miura transformations,

- have particular solutions in terms of special functions or rational solutions for special values of their parameters. These solutions can be written in terms of Casorati determinants, and

- can be cast into bilinear forms. 
And now we come to the crucial question of d-P $\mathrm{P}_{\mathrm{VI}}$. Clearly a non-autonomous threepoint mapping of the QRT type must exist, having $\mathrm{P}_{\mathrm{VI}}$ as its continuous limit. It would be just the deautonomization of the generic mapping (3.4) presented above. (This is expected to be true in particular in view of our remark in Section 3.1, concerning the fact that the only autonomous mapping of the form (3.4) obtained through singularity confinement was the QRT one.) Still, the computations leading to an explicit form of the coefficients are prohibitively cumbersome, and we cannot give the precise form of $\mathrm{d}-\mathrm{P}_{\mathrm{VI}}$. We shall content ourselves here to a plausibility argument based on the structure of the singularities of the known d-Ps. For the d-Ps presented above, we have the following singularity patterns.

The discrete $\mathrm{d}-\mathrm{P}_{\mathrm{V}}$ has 8 singularities $x^{(1)}, \ldots, x^{(6)}$ and $\infty$ (double). They are organized as follows: $x^{(1)}$ goes over to $x^{(2)}$ in one step, and vice versa, which we will simply denote as $\left\{x^{(1)}, x^{(2)}\right\},\left\{x^{(2)}, x^{(1)}\right\}$, and similarly $\left\{x^{(3)}, x^{(4)}\right\},\left\{x^{(4)}, x^{(3)}\right\}$. (Clearly these singularities correspond to $a, 1 / a, b, 1 / b$, that appear in the numerator of the left-hand side of (3.32), and the fact that $x^{(2)}=1 / x^{(1)}, x^{(4)}=1 / x^{(3)}$ is an extra integrability requirement.) Finally, the remaining two finite singularities (to be identified with $p, q$ in the denominator) follow the pattern $\left\{x^{(5)}, \infty, x^{(6)}\right\}$ and $\left\{x^{(6)}, \infty, x^{(5)}\right\}$. For $\mathrm{d}-\mathrm{P}_{\mathrm{IV}}$, we have the same structure. The 8 singularities are now identified in the following way: $x^{(1)}=a, x^{(2)}=-a, x^{(3)}=b, x^{(4)}=-b, x^{(5)}=c-z$, $x^{(6)}=-c-z$, and $\infty$ (double). For d-P III, the $f_{i}$ s are cubic and the singularities are $x^{(1)}, \ldots, x^{(4)}, \infty$ (double), and 0 (double), where the $x^{(i)}$ 's must be identified with the quantities $a, b, c, d$ that appear in the equation. The singularity patterns are $\left\{x^{(1)}, \infty, x^{(2)}\right\},\left\{x^{(2)}, \infty, x^{(1)}\right\},\left\{x^{(3)}, 0, x^{(4)}\right\}$, and $\left\{x^{(4)}, 0, x^{(3)}\right\}$. In the case of d-P II, we have $f_{3}=0, f_{1}$ and $f_{2}$ quadratic, and thus in principle only four singularities, $x^{(1)}$, $x^{(2)}$, and $\infty$ (double). The $x^{(1)}, x^{(2)}$ are traditionally set to \pm 1 , and we obtain the patterns $\{1, \infty,-1\}$ and $\{-1, \infty, 1\}$. Finally, for $d-P_{I}$, the singularities are 0 and $\infty$, (both double). We obtain the singular pattern $\{0, \infty, \infty, 0\}$.

The discrete $\mathrm{P}_{\mathrm{VI}}$, since it is the analog of $\mathrm{P}_{\mathrm{VI}}$, i.e., the generic and most symmetric among the Painlevé equations, would be expected to have 8 distinct singularities (three of which can be taken equal to 0,1 , and $\infty$ ). In analogy to the autonomous case, we expect its singularity patterns to be $\left\{x^{(1)}, x^{(2)}\right\},\left\{x^{(2)}, x^{(1)}\right\},\left\{x^{(3)}, x^{(4)}\right\},\left\{x^{(4)}, x^{(3)}\right\}$, $\left\{x^{(5)}, x^{(6)}\right\},\left\{x^{(6)}, x^{(5)}\right\},\left\{x^{(7)}, x^{(8)}\right\},\left\{x^{(8)}, x^{(7)}\right\}$. (In Section 3.1, we have paired the singularities in a slightly different way, $\left\{x^{(i)}, x^{(i+4)}\right\}$, but, clearly, this is just a question of renaming.) Still, as explained above, the explicit implementation of the confinement conditions leads to equations for the $x^{(i)}(n)$ in terms of the $x^{(i)}\left(n_{0}\right)$ and $x^{(i)}\left(n_{0}+1\right)$ in an explicit way. This system of equations, however, is much more complicated than $\mathrm{P}_{\mathrm{VI}}$ itself, and no explicit solutions are yet available.

This completes our list of mappings that are the discrete equivalent of the PainlevéGambier equations.

\section{Conclusion and perspectives}

In this paper, we have tried to establish the equivalent of the Gambier classification for discrete systems in the form of three-point mappings. As we have shown in the previous sections, the parallel is perfect (although differences between discrete and continuous systems do exist). Thus, examples of mappings can be presented for all three integrable classes: integrable through quadratures, linearizable, and Painlevé 
transcendents. The study of the properties of these discrete equations and of their solutions as well as the problem of their classification define whole new directions of research which we have just started exploring.

Several questions remain open at this moment concerning the discrete analogs of integrable differential equations. In this study, we have limited ourselves to equations of the first and second order explicit in the highest derivative. However, there exists a class of equations where the highest derivative enters through some power larger than one: the Briot-Bouquet first-order equations [24] $\left(w^{\prime}\right)^{n}=f(w, z)$ and the equations studied by Bureau and Cosgrove [7] at second order of the form $\left(w^{\prime \prime}\right)^{n}=f\left(w^{\prime}, w, z\right)$. Their discrete analogs have never been studied (at least to the authors' knowledge). Even without resorting to nonlinearities of the highest derivative, equations in the form of a system of two two-point mappings have not been studied in any generality. Their study is all the more interesting (and difficult) since several examples of integrable mappings $x_{n+1}=f\left(x_{n}, y_{n}, n\right), y_{n+1}=g\left(x_{n}, y_{n}, n\right)$ do exist [11]. Moreover, the guide of the continuous limit does not exist here, since the study of continuous systems $w^{\prime}=f(w, u, z), u^{\prime}=g(w, u, z)$ is far from complete. The same is true for third-order equations $w^{\prime \prime \prime}=f\left(w^{\prime \prime}, w^{\prime}, w, z\right)$. The study of the corresponding 4-point mappings is only schematic at this moment, although it is of a considerable interest: in fact, in [29], Nijhoff has shown that a natural form of a $q$-deformed $\mathrm{d}-\mathrm{P}_{\mathrm{I}}$ is the 4-point equation

$$
\beta q^{-n}\left(x_{n+1}-q^{2} x_{n-2}\right)=q \frac{(n)_{q}+\alpha q^{n}}{x_{n}}-\frac{(n-1)_{q}+\alpha q^{n-1}}{x_{n-1}}
$$

where $(n)_{q}=\left(q^{n}-1\right) /(q-1)$. The discrete analog of higher-order equations also would be of great interest since it would allow us to obtain discrete forms for well-known integrable hamiltonian systems [42].

Moving away from the purely discrete systems, one encounters further interesting problems. The domain of differential-difference equations has only been touched upon in our work in [19]. Still, this first study revealed the existence of the delay-differential equivalents to the Painleve equations. Here also, there exist several possibilities combining higher order derivatives with higher order mappings. We expect the equations that will result from such studies (and the ones mentioned in the previous paragraphs) to be much richer than the ones discovered one century ago by Painlevé and Gambier. It would be even more satisfactory if they turned out to have the vast domain of physical applications that the Painlevé equations enjoy today.

Acknowledgements. The authors wish to express their gratitude to all who have assisted them over the years in exploring the new world of discrete integrable systems either through direct collaboration or through discussions and exchange of correspondence: M. J. Ablowitz, F. Bureau, H. Capel, P. Clarkson, B. Dorizzi, A. Fokas, J. Hietarinta, R. Hirota, N. Joshi, K. Kajiwara, G. Karra, Y. Kosmann-Schwarzbach, M. D. Kruskal, D. Levi, B. McCoy, J-M. Maillard, I. Moreira, U. Mugan, F. Nijhoff, Y. Ohta, V. Papageorgiou, G. Rollet, J. Satsuma, J-M. Strelcyn, K. M. Tamizhmani, and P. Winternitz. 


\section{References}

1. M. J. Ablowitz and J. Ladik, Nonlinear differential-difference equations, J. Math. Phys. 16 (1975), 598-603.

2. M. J. Ablowitz, A. Ramani, and H. Segur, Nonlinear evolution equations and ordinary differential equations of Painlevé-type, Lett. Nuov. Cim. 23 (1978), 333-338.

3. M. J. Ablowitz and H. Segur, Asymptotic solutions of the Korteweg-de Vries equation, Stud. Appl. Math. 57 (1977), 13-44.

4. __ Exact linearization of Painlevé transcendents, Phys. Rev. Lett. 38 (1977), 1103-1106.

5. R. J. Baxter, Exactly Solvable Models in Statistical Mechanics, Academic Press, London, 1982.

6. E. Brézin and V. A. Kazakov, Exactly solvable field theories of closed strings, Phys. Lett. 236B (1990), 144-149.

7. F. Bureau, Equations différentielles du second ordre en $Y$ et du second degré en $\ddot{Y}$ dont l'intégrale est à points critiques fixes, Ann. di Math. 91 (1972), 163-172.

8. C. M. Cosgrove, All binomial-type Painlevé equations of the second order and degree three or higher, Stud. Appl. Math. 90 (1993), 119-187.

9. __ Corrections and annotations to Ince's Chapter 14.

10. E. Fermi, J. Pasta, and S. Ulam, Studies of nonlinear problems, Los Alamos report LA1940 (1955).

11. A. S. Fokas, B. Grammaticos, and A. Ramani, From continuous to discrete Painlevé equations, J. Math. Anal. and Appl. 180 (1993), 342-360.

12. B. Gambier, Sur les équations différentielles du second ordre et du premier degré dont l'intégrale est à points critiques fixes, Acta Math. 33 (1910), 1-55.

13. C. S. Gardner, J. M. Greene, M. D. Kruskal, and R. M. Miura, Method for solving the Kortewegde Vries equation, Phys. Rev. Lett. 19 (1967), 1095-1097.

14. R. Garnier, Sur les équations différentielles du troisième ordre dont l'intégrale générale est uniforme et sur une classe d'eq́uations nouvelles d'ordres supérieurs dont l'intégrale générale a ses points critiques fixes, Ann. ScI. Ec. Norm. Sup. 29 (1912), 1-126.

15. B. Grammaticos and B. Dorizzi, Integrable discrete systems and numerical integrators, J. Math. Comp. in Sim. 37 (1994), 341-352.

16. B. Grammaticos, F. W. Nijhoff, V. Papageorgiou, A. Ramani, and J. Satsuma, Linearization and solution of the discrete Painlevé-III equation, Phys. Lett. A185 (1994), 446-452.

17. B. Grammaticos and A. Ramani, The Gambier mapping, Physica A223 (1995), 125-136.

18. _ Discrete Painlevé equations: derivation and properties, NATO ASI C413 (1993), 299313.

19. B. Grammaticos, A. Ramani, and I. C. Moreira, Delay differential equations and the Painlevé transcendents, Physica A196 (1993), 574-590.

20. B. Grammaticos, A. Ramani, and V. G. Papageorgiou, Do integrable mappings have the Painlevé property?, Phys. Rev. Lett. 67 (1991), 1825-1828.

21. B. Grammaticos, A. Ramani, and K. M. Tamizhmani, Nonproliferation of preimages in integrable mappings, Jour. Phys. A27 (1994), 559-566.

22. S. P. Hastings and J. B. McLeod, A boundary-value problem associated with the second Painlevé transcendent and the Korteweg-de Vries equation, Arch. Rat. Mech. Anal. 73 (1980), 31-51.

23. R. Hirota, Discrete analogue of a generalized Toda equation, J. Phys. Soc. Jpn 50 (1981), 37853791.

24. E. L. Ince, Ordinary differential equations, Dover, New York, 1956.

25. M. Jimbo and T. Miwa, A study of holonomic fields, Proc. Japan Acad. 56A (1980), 405-410.

26. M. Jimbo, T. Miwa, and K. Ueno, Monodromy preserving deformation of linear ordinary differential equations, Physica D2 (1981), 306-352.

27. P. D. Lax, Integrals of nonlinear equations of evolution and solitary waves, Comm. Pure and Appl. Math 21 (1968), 467-490.

28. B. M. McCoy and F. Y. Wu, Nonlinear partial difference equations for the two-dimensional Ising model, Phys. Rev. Lett. 45 (1980), 675-679.

29. F. W. Nijhoff, On a q-deformation of the discrete Painlevé I equation and q-orthogonal polynomials, Lett. Math. Phys. 30 (1994), 327-336. 
30. F. W. Nijhoff and V. G. Papageorgiou, Similarity reductions of integrable lattices and discrete analogues of the Painlevé-II equation, Phys. Lett. 153A (1991), 337-343.

31. F. W. Nijhoff, V. Papageorgiou, and H. W. Capel, Integrable time-discrete systems: lattices and mappings, Springer Lect. Notes in Math. 1510 (1992), 312-325.

32. P. Painlevé, Sur les équations différentielles du second ordre et d'ordre supérieur dont l'intégrale générale est uniforme, Acta Math. 25 (1902), 1-85.

33. V. Periwal and D. Shevitz, Unitary-matrix models as exactly solvable strings theories, Phys. Rev. Lett. 64 (1990), 1326-1329.

34. Proceedings of the centennial KdV conference, Acta Applic. Math. 39 (1995).

35. G. R. W. Quispel, F. W. Nijhoff, H. W. Capel, and J. van der Linden, Linear integral equations and nonlinear differential-difference equations, Physica A125 (1984), 344-380.

36. G. R. W. Quispel, J. A. G. Roberts, and C. J. Thompson, Integrable mappings and soliton equations, Physica D34 (1989), 183-192.

37. A. Ramani and B. Grammaticos, The hunting for the $d-P_{V I}$, in preparation.

38. A. Ramani, B. Grammaticos, and A. Bountis, The Painlevé property and singularity analysis of integrable and non-integrable systems, Phys Rep 180 (1989), 159-245.

39. A. Ramani, B. Grammaticos, and J. Hietarinta, Discrete versions of the Painlevé equations, Phys. Rev. Lett. 67 (1991), 1829-1832.

40. A. Ramani, B. Grammaticos, and G. Karra, Linearizable mappings, Physica A181 (1992), 115127.

41. A. Ramani, B. Grammaticos, and V. G. Papageorgiou, Singularity confinement, CRM Proc. and Lect. Notes 9 (1996), 303-318.

42. Yu. B. Suris, Discrete-time analogs of some nonlinear oscillators in the inverse-square potential, Jour. Phys. A27 (1994), 8161-8170.

43. N. J. Zabusky and M. D. Kruskal, Interaction of solitons in a collisionless plasma and the recurrence of initial states, Phys. Rev. Lett. 15 (1965), 240-243.

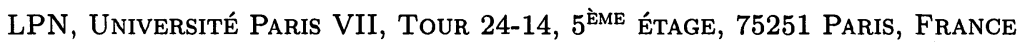

CPT, Ecole Polytechnique, CNRS, UPR 14, 91128 Palaiseau, France 